\title{
Observation of a higher-order topological bound state in the continuum
}

\author{
Alexander Cerjan ${ }^{*}$ Marius Jürgensen, Wladimir A. Benalcazar, Sebabrata Mukherjee, and Mikael C. Rechtsman† \\ Department of Physics, The Pennsylvania State University, University Park, Pennsylvania 16802, USA
}

(Dated: June 12, 2020)

\begin{abstract}
Higher-order topological insulators are a recently discovered class of materials that can possess zero-dimensional localized states regardless of the dimension of the lattice. Here, we experimentally demonstrate that the topological corner-localized modes of higher-order topological insulators can be symmetry protected bound states in the continuum; these states do not hybridize with the surrounding bulk states of the lattice even in the absence of a bulk bandgap. As such, this class of structures has potential applications in confining and controlling light in systems that do not support a complete photonic bandgap.
\end{abstract}

Topological materials have garnered significant interest for their ability to support boundary-localized states that manifest exotic phenomena, such as the backscatterfree chiral edge states found in Quantum Hall systems [1 14], and edge-localized states found in systems with quantized dipole moments [15-17]. Recently, it was discovered that crystalline symmetries can give rise to a new class of materials with topological phases that can protect zero-dimensional corner-localized states in two dimensions, or more generally $d-n$ dimensional states at the boundaries of $d$ dimensional lattices, with $n \geq 2$ [1832. Coined higher-order topological insulators, if these systems are also chiral or particle-hole symmetric, their corner-localized states appear at the center of their energy spectrum. However, the crystalline symmetries that protect these phases do not necessitate the formation of a bulk bandgap in the middle of the spectrum. This raises an intriguing question, namely, do these states remain long-lived in-band resonances, exponentially localized to the corner when they are degenerate with the surrounding bulk bands, or do they instead hybridize with the bulk states and lose their spatial localization? If the state remains fully spatially localized to the corner forever, it is a bound state in the continuum (BIC) [33 40, where the continuum is formed by the surrounding bulk bands whose states extend throughout the infinite lattice; otherwise the state becomes a standard resonance, where any energy initially added to the corner will eventually radiate away into the infinite lattice with finite lifetime. Recently, it was theoretically predicted that a higherorder topological phase can be used to protect a cornerlocalized BIC if the system satisfies additional symmetry requirements 41.

Finding a protected zero-dimensional BIC in a material whose topology is only dependent upon the crystalline symmetries of the lattice presents a significant opportunity in the context of two- and three-dimensional photonic crystals 42. Such states could be used to realize cavities in low-index photonic crystals, where there are no known crystal geometries which yield complete bandgaps 43, 44]. However, although higher-order

\footnotetext{
* awc19@psu.edu

$\dagger$ mcrworld@psu.edu
}

topological phases have now been demonstrated in a wide range of different physical platforms, including microwaves 45, photonics 46 48, acoustics 49 54, electric circuits [55, 56, and atomic systems [57], all of these previous studies have been limited to insulator-like systems, and exhibit their corner-localized states spectrally isolated from their surrounding bulk bands.

Here, we experimentally realize a higher-order topological bound state in the continuum using a twodimensional waveguide array comprised of evanescentlycoupled waveguides [58, 59]. To show that our waveguide array possesses a BIC, we perform three separate experiments. First, by injecting light into the corner of the array, we prove that the lattice exhibits a corner-bound mode when the lattice is in its topological phase, and that this mode disappears across the topological phase transition. Second, by using an auxiliary waveguide to couple into the array, which fixes the effective energy of the initial excitation, we show that this corner-localized mode appears at zero energy, and is degenerate with bulk states of the lattice. For consistency with previous studies, we refer to 'zero energy' as the propagation constant / energy of a single waveguide, which for chiral-symmetric lattices is at the center of the spectrum. Finally, we show that our bound state transforms into a resonance when we break chiral symmetry by detuning the index of refraction of the members of one sublattice. Together, these experiments prove that the corner-localized state of our higher-order topological waveguide array is a symmetryprotected BIC, and does not hybridize with the bulk bands so long as the necessary symmetries remain intact.

Our experimental array consists of a square lattice in which each unit cell contains four waveguides and is $C_{4 v}$ symmetric, as shown in Fig. 1, b 28, 41, 60, 61. As each waveguide within the lattice only supports a single bound mode for the wavelengths we consider, and the coupling between waveguides decreases exponentially with increasing separation, our waveguide array can be approximated using a tight-binding model with only nearest-neighbor couplings, such that the lattice is chiral (sublattice) symmetric. The diffraction of light through the structure is governed by

$$
i \partial_{z}|\psi(z, \lambda)\rangle=\hat{H}(\lambda)|\psi(z, \lambda)\rangle
$$

Here, $|\psi(z, \lambda)\rangle$ is the envelope of the electric field on each 

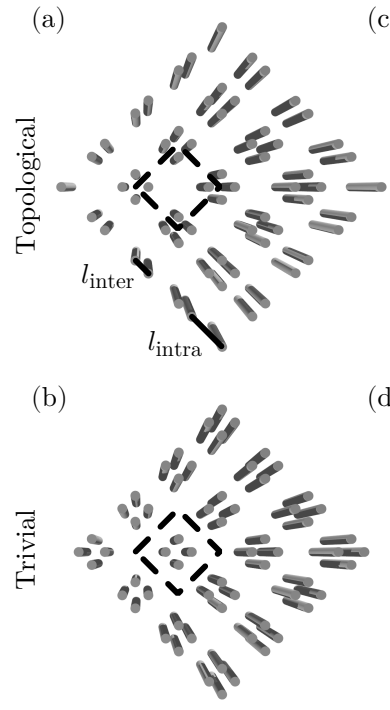

(c)

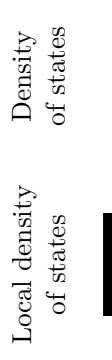

(d)

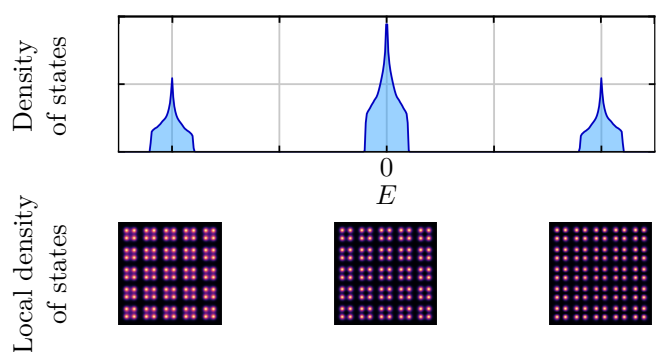

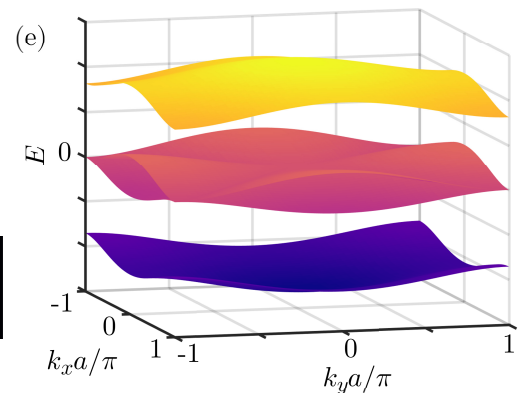

(f)

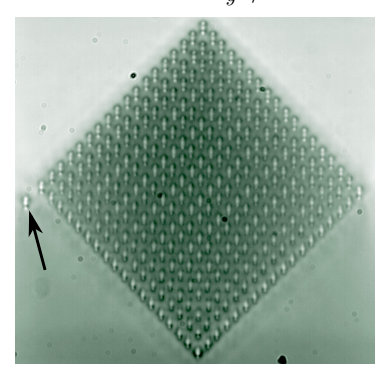

FIG. 1. Higher-order topological insulator in a waveguide array. (a),(b) Schematic of a higher-order topological insulator in its topological (a) or trivial (b) phase. The unit cell of each phase is indicated in the dashed-black square. The distances between adjacent waveguides within a unit cell, and between neighboring unit cells, are shown as $l_{\text {intra }}$ and $l_{\text {inter }}$, respectively. Schematics are not to scale. (c),(d) Density of states (top panel) and associated local density of states (bottom panel) for each band for the topological and trivial phases of the higher-order topological insulator in finite geometries, respectively. The density of states is calculated using the tight-binding approximation. (e) Bulk band structure for the higher-order topological insulator, which is identical for both the topological and trivial phases, calculated using full-wave numerical simulations for $\lambda=850 \mathrm{~nm}, l_{\text {intra }}=17 \mu \mathrm{m}$, and $l_{\text {inter }}=13 \mu \mathrm{m}$. (f) White light transmission micrograph of the output facet of a waveguide array with $l_{\text {intra }}=13 \mu \mathrm{m}$ and $l_{\text {inter }}=11 \mu \mathrm{m}$. An auxiliary waveguide into which light can be injected, $20 \mu \mathrm{m}$ away from the array, is indicated with a black arrow.

of the waveguides at propagation distance $z$ and wavelength $\lambda$. The coupling coefficients, $t_{\text {intra }}$ and $t_{\text {inter }}$, in $\hat{H}$ are determined by the spacings between neighboring waveguides within the same unit cell, $l_{\text {intra }}$, and between adjacent unit cells, $l_{\text {inter }}$.

For an array which is infinite in the transverse plane, the Bloch Hamiltonian of the lattice can be written as

$$
\begin{gathered}
h\left(k_{x}, k_{y}\right)=\left(\begin{array}{cc}
0 & Q \\
Q^{\dagger} & 0
\end{array}\right), \\
Q=\left(\begin{array}{cc}
t_{\text {intra }}+t_{\text {inter }} e^{i k_{x} a} & t_{\text {intra }}+t_{\text {inter }} e^{i k_{y} a} \\
t_{\text {intra }}+t_{\text {inter }} e^{-i k_{y} a} & t_{\text {intra }}+t_{\text {inter }} e^{-i k_{x} a}
\end{array}\right),
\end{gathered}
$$

in which $a$ is the lattice constant. To assist with comparisons with the topological literature, we will refer to the eigenvalues of the waveguide array, $\hat{H}$, as energies, $E$, while noting that physically these values correspond to shifts in momentum, $\beta=-E=k_{z}-k_{0}$, of $|\psi\rangle$ along the $z$ axis. Here, $k_{0}=\omega n_{0} / c$, where $n_{0}$ is the index of refraction of the borosilicate glass into which the waveguides are fabricated and $\omega$ is the frequency of the injected light. As all of these modes are bound modes of the waveguides, 'zero energy' refers to the energy at the middle of this spectrum.

The presence of $C_{4 v}$ symmetry permits two distinct topological phases depending on the ratio of the relative spacings between neighboring waveguides within and between adjacent unit cells. When the lattice is in its topo- logical phase, with $l_{\text {intra }} / l_{\text {inter }}>1$, the bands possess different representations of $C_{4 v}\left(C_{2 v}\right)$ at the corresponding high-symmetry points in the Brillouin Zone, $\mathbf{M}(\mathbf{X}$ and $\mathbf{Y})$, than at $\boldsymbol{\Gamma}$. However, when the lattice is in its trivial phase, with $l_{\text {intra }} / l_{\text {inter }}<1$, the bands possess the same symmetry representation at all of the highsymmetry points. The topological phase transition occurs at $l_{\text {intra }} / l_{\text {inter }}=1$, when the bulk bandgap closes at the high-symmetry points, allowing for the exchange of their representations of these crystalline symmetries. In a finite lattice, these two phases can be distinguished by their density of states, as well as the associated local density of states of each band, shown in Fig. 1k,d. In its topological phase, this lattice exhibits both edgelocalized states in its bulk bandgaps protected by $C_{2}$ symmetry, as well as a corner-induced filling anomaly also protected by $C_{2}$ 28, 41]. In Fig. 1;, the presence of these extra corner-localized states can be observed in the local density of states of the central bulk band of the lattice. Note that when $l_{\text {intra }}$ is interchanged with $l_{\text {inter }}$, both the topological and trivial phases of the array have the same bulk band structure consisting of four bands, as displayed in Fig. 17. An example of a facet of a waveguide array is shown in Fig. 1f.

In the presence of $C_{4 v}$ and chiral symmetries, the lattice will always have gapless bulk bands at zero energy, regardless of its topological phase. These same two sym- 

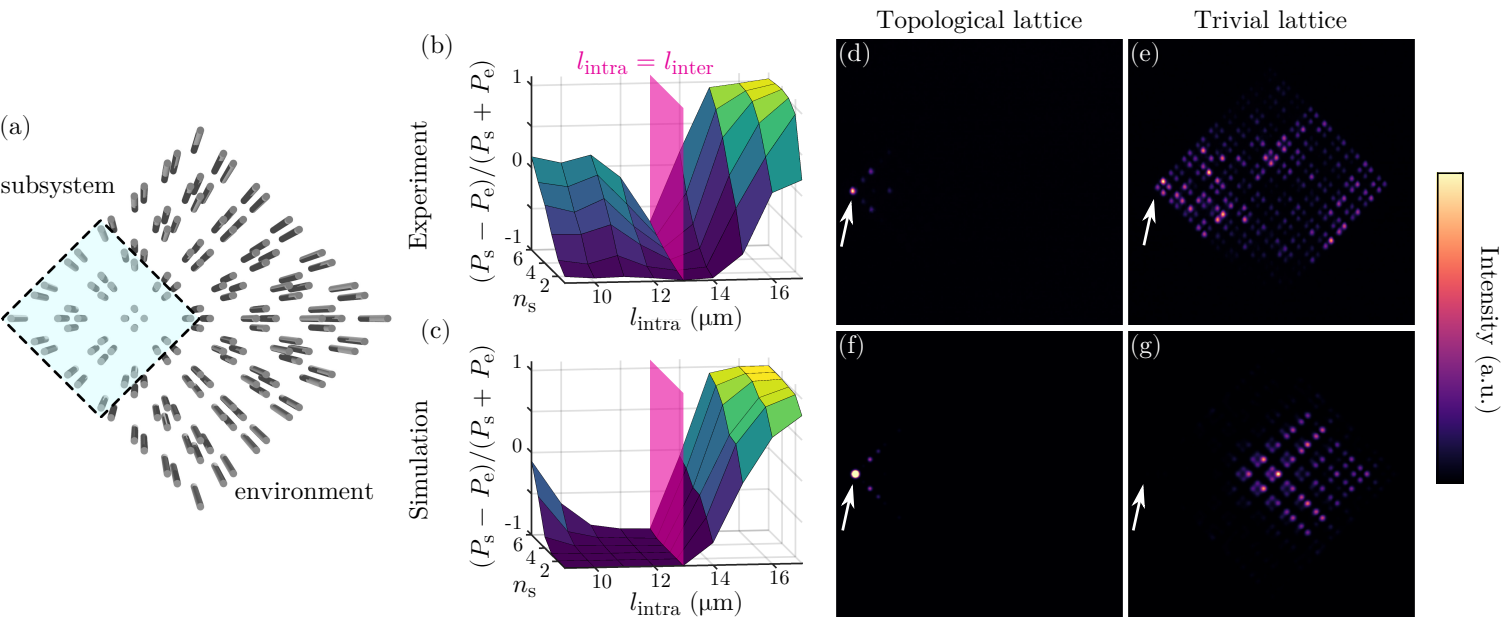

FIG. 2. Bound state in a higher-order topological insulator. (a) Schematic of a waveguide array in the topological phase with the boundary of the 'subsystem' for $n_{\mathrm{s}}=3$ indicated. (b),(c) Experimentally observed (b) and numerically simulated (c) fractional power, as a function of the size of the subsystem, $n_{\mathrm{s}}$, and the spacing between adjacent waveguides within the same unit cell, $l_{\text {intra }}$. Spacing between adjacent waveguides in neighboring unit cells is fixed at $l_{\text {inter }}=13 \mu \mathrm{m}$, and the wavelength of the light is $\lambda=850 \mathrm{~nm}$. The maximum propagation distance of the array is $L=7.6 \mathrm{~cm}$. The location of the topological transition is denoted as a magenta plane at $l_{\text {intra }}=l_{\text {inter }}=13 \mu \mathrm{m}$. (d) Experimentally observed intensity at the output facet for $l_{\text {intra }}=17 \mu \mathrm{m}$. Light is injected into the left-most waveguide at the corner of the array, marked with a white arrow. (e) Experimentally observed intensity at the output facet for $l_{\text {intra }}=9 \mu \mathrm{m}$. (f)-(g) Same as (d)-(e), except for full wave numerical simulations of the waveguide array.

metries also pin the corner-localized states to zero energy, guaranteeing that the states will always be degenerate with the bulk bands of the array, while simultaneously protecting the corner-localized modes from hybridizing with the surrounding bulk states 41 . This protection comes in two parts. First, two combinations of the four corner states have incompatible symmetry representations with those of the surrounding bulk bands at zero energy, and thus cannot hybridize with them. Then, the two remaining combinations of corner localized states must be both rotationally symmetric partners, with the same energy, and chiral symmetric partners, with opposite energies, forcing their energies to remain pinned at zero. This prevents these two corner-localized states from hybridizing with the degenerate bulk states to change their energies or modal profiles, and as such any hybridization of the corner states with the surrounding bulk states is simply a change in basis that does not alter their underlying spatially-localized nature. This means that all four corner states in our lattice are topologically guaranteed to be zero-dimensional symmetry-protected bound states in the continuum.

In our experiment, it is not possible to completely remove the next-nearest-neighbor couplings which exist between the waveguides in the array, which means our lattice is not perfectly chiral symmetric. However, the decay length of the corner state due to this slight symmetry breaking $(\sim 25 \mathrm{~m})$ is significantly longer than the propagation length in our experiments, $L=7.6 \mathrm{~cm}$, see Supplementary Information. As such, our experimental array is effectively chiral symmetric.

Previous studies of systems supporting BICs have iden- tified that BICs can also be protected by separability, i.e. that the Hamiltonian of the system can be divided into two independent sectors, $\hat{H}(\mathbf{r})=\hat{H}_{x}(x)+\hat{H}_{y}(y)$ 34. However, despite the fact that Eqs. (2)-(3) are separable, separability is not what protects the higher-order topological BICs we consider here. Analytically, one still observes higher order topological BICs when additional terms have been added to the lattice's Hamiltonian which obey $C_{4 v}$ and chiral symmetries but break separability [41, see Supplementary Information.

To experimentally prove that our waveguide array contains a higher-order topological BIC, we first inject light into the corner of the array, and observe whether most of the light remains confined to this corner or diffracts into the bulk. To assess the localization of the light at the output facet of the array, we divide the array into two regions, the 'subsystem' which represents the square of unit cells with side length $n_{\mathrm{s}}$ closest to the corner, as indicated in Fig. 2a, while the remaining waveguides comprise the 'environment.' This terminology is chosen for consistency with previous studies of bound states in the continuum, in which the subsystem (where light is confined) and its surrounding radiative environment are typically physically distinct regions containing different types of structures. We then compare the total output power observed in the subsystem, $P_{\mathrm{s}}$, with that observed in the environment, $P_{\mathrm{e}}$, using the figure of merit $\left(P_{\mathrm{s}}-P_{\mathrm{e}}\right) /\left(P_{\mathrm{s}}+P_{\mathrm{e}}\right)$. For this 'fractional power,' values near +1 correspond to all of the output power being localized in the subsystem, while values of -1 indicate that all of the output power has diffracted into the environment. In Figs. 2b,c, we show the fractional power as a 

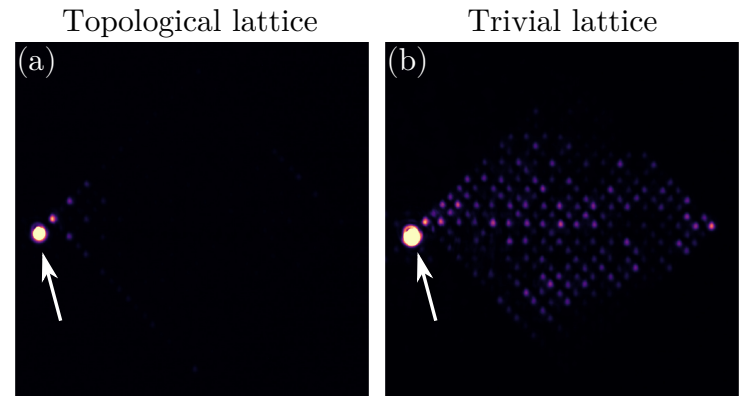

FIG. 3. Observation of the BIC and surrounding continuum in a higher-order topological insulator. (a) Experimentally observed intensity at the output facet for a topological waveguide array, with $l_{\text {intra }}=13 \mu \mathrm{m}$ and $l_{\text {inter }}=11 \mu \mathrm{m}$. Light is injected into the array at $\lambda=900 \mathrm{~nm}$ using an auxiliary waveguide placed $20 \mu \mathrm{m}$ away from the corner of the lattice (marked with a white arrow). The total length of the array is $L=7.6 \mathrm{~cm}$. (b) Experimentally observed intensity at the output facet for a trivial waveguide array, with $l_{\text {intra }}=11 \mu \mathrm{m}$ and $l_{\text {inter }}=13 \mu \mathrm{m}$.

function of the topological phase of the array, $l_{\text {intra }} / l_{\text {inter }}$, as well as the the size of the subsystem, $n_{\mathrm{s}}$. Here, we can clearly see that the light remains localized to the subsystem, regardless of its size, until the arrays approach the topological phase transition, at $l_{\text {intra }} / l_{\text {inter }}=1$. The observed intensity at the output facet is shown for an example of both the topological and trivial arrays in Figs. $2 \mathrm{~d}$-g. Note that the increase seen in the fractional power for large subsystem sizes for some topologically trivial arrays is due to spurious reflections off of some of the waveguides at the top and bottom of the array, as well as back-reflections off of the far side of the array. Nevertheless, it is clear from Fig. 2 e that these arrays do not possess a bound state. Thus, these results indicate that the topological waveguide array possesses a bound state that is connected to the topological phase of the lattice, but does not yet prove that the bound state is degenerate with the surrounding bulk bands.

To prove that this topological bound state is a BIC, we use a waveguide array with an auxiliary waveguide weakly coupled to the lattice and placed near one of the corners. Since this waveguide is identical to all others in the lattice, it effectively acts as a fixed zero-energy source. All light injected into it can only excite states near zero-energy in the array. As can be seen in Fig. 3a, when the waveguide array is in its topological phase and the auxiliary waveguide is placed near a corner of the lattice, the dominant excited mode of the waveguide array is the topological corner-localized mode. However, when $l_{\text {intra }}$ and $l_{\text {inter }}$ are reversed, the bulk of the lattice remains completely unchanged, but the array is now in the trivial phase. Upon excitation using an auxiliary waveguide, we see that bulk states of the lattice are excited, and there is no corner-localized mode. Since the lattice bulk is identical in both cases, we can conclude that there are zero-energy bulk states that are degenerate with the corner-localized mode in the topological case. This experimentally proves that the corner-localized topological bound states in this array are BICs.

Finally, to demonstrate that this higher-order topological BIC is protected by chiral symmetry, we purposefully break chiral symmetry by increasing the refractive index on two of the four waveguides in the unit cell, as indicated in Fig. 4 a, which has the effect of decreasing the effective on-site energy of these two lattice sites. This has several effects on the array. First, this opens a bulk bandgap in the center of the spectrum, in which one of the two central bulk bands remains at 'zero-energy' (which is no longer at the middle of the spectrum), while the other's energy decreases, as shown in Fig. $4 \mathrm{p}$. Second, as the modal profile of each corner-localized state is only supported on two of the four waveguides in the unit cell (diagonally across from one another), this change also breaks the four-fold degeneracy of the corner-localized states. Instead, the pair of corner-localized states whose modal profiles overlap with the perturbation decrease their energy, remaining degenerate with the higher of the two central bulk bands, while the other pair of cornerlocalized states remain degenerate with the bulk band at 'zero energy' (which is now not the center of the spectrum). This can be seen in the local density of states for each band of the array, shown in Fig. 4k. However, now that chiral symmetry has been broken, the cornerlocalized modes are allowed to hybridize with states from their respective bulk bands, transforming from BICs into resonances of the lattice. We can observe this transition of one of the BICs into a resonance by incrementally increasing the strength of the sublattice symmetry breaking, and coupling into the lattice using an auxiliary waveguide, which remains at zero energy, as shown in Figs. 4h-h. As chiral symmetry is lost, as in Figs. 4a-h, the wavefunction within the array begins to disassociate from the corner, and the maximum of this wavefunction travels into the bulk of the array and along the edges, signifying that all of the states that are being excited by the auxiliary waveguide have significant spatial overlap with the other modes of the lattice. In other words, the corner-localized state has become a resonance and is no longer a BIC. This is in clear contrast to what is seen in Fig. $4 \mathrm{~d}$, where chiral symmetry is intact and the wavefunction in the lattice remains localized to the corner, indicating the presence of a BIC.

In conclusion, we have experimentally observed a higher-order topological bound state in the continuum in a waveguide array. This BIC is protected by $C_{4 v}$ and chiral symmetries, and is topologically guaranteed to exist at zero energy in the lattice. Moreover, as these states are able to confine light to a zero-dimensional mode in the absence of a bulk bandgap, which is a greater reduction in dimensionality than is found in other systems supporting BICs [34, they are a promising candidate for creating cavities in low-index photonic platforms in the absence of a complete photonic bandgap. Current designs for photonic crystals that support band gaps require refractive 
(a)

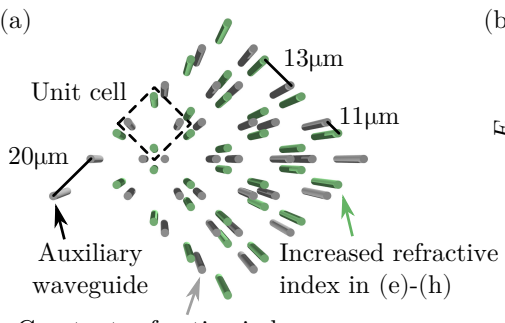

Constant refractive index

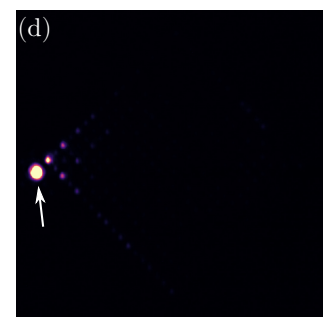

Chiral symmetric (b)
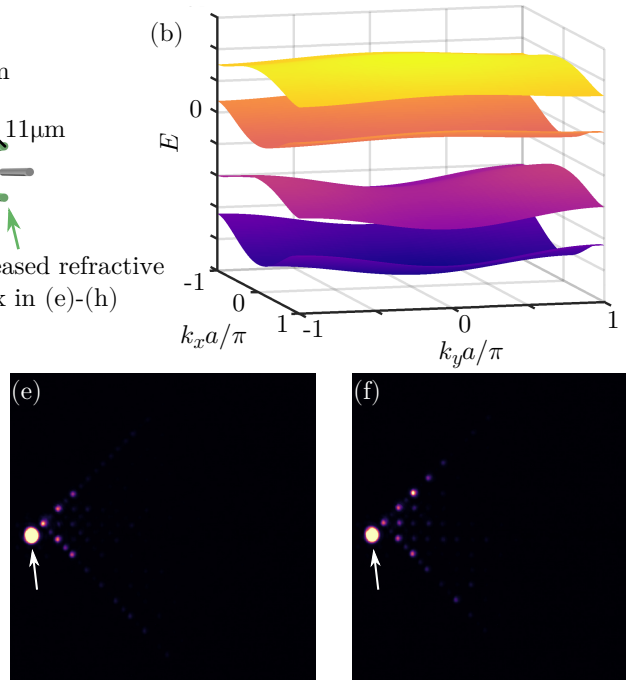

Increasing chiral symmetry breaking (c)
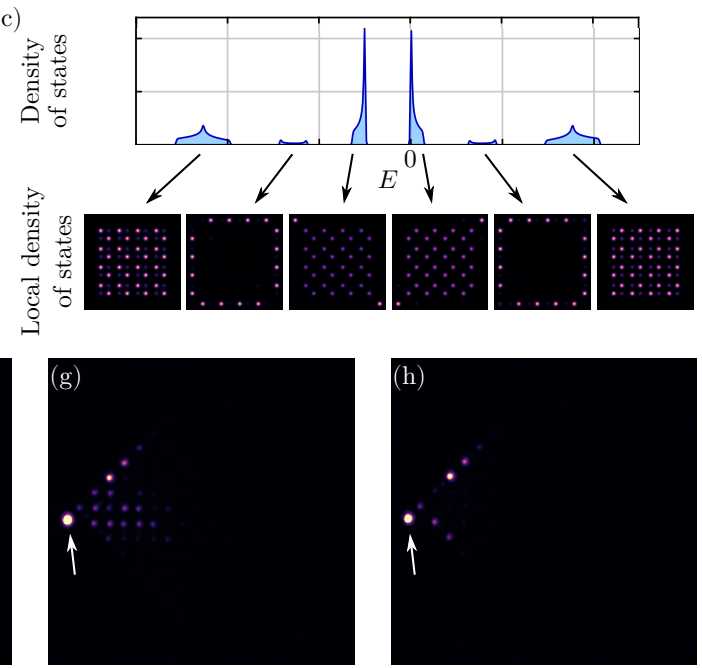

FIG. 4. Observation of a BIC turning into a resonance as chiral symmetry is broken by detuning the sublattices of the lattice. (a) Schematic of a higher-order topological waveguide array with broken chiral symmetry. The auxiliary waveguide where light is initially injected is $20 \mu \mathrm{m}$ away from the waveguide array. The waveguides colored in green have been fabricated using slower writing speeds, resulting in a larger refractive index, and thus a decreased on-site energy. (b) Bulk band structure for the higher-order topological insulator with broken chiral symmetry, calculated using full-wave numerical simulations for $\lambda=900 \mathrm{~nm}$, $l_{\text {intra }}=13 \mu \mathrm{m}, l_{\text {inter }}=11 \mu \mathrm{m}$, and in which two of the waveguides have an increased refractive index, $\Delta n_{0}=3.3 \cdot 10^{-3}$, as opposed to $\Delta n_{0}=2.9 \cdot 10^{-3}$. These parameters correspond to the experimental results shown in (f), below. (c) Density of states (top panel) and associated local density of states for each band (bottom panel) for a tight-binding lattice with broken chiral symmetry. Zero energy (center of the spectrum) of the chiral symmetric (unperturbed) array is marked. (d) Experimentally observed intensity at the output facet of the symmetric waveguide array, with the same refractive index on all of the waveguides, with $l_{\text {intra }}=13 \mu \mathrm{m}$ and $l_{\text {inter }}=11 \mu \mathrm{m}$, for an incident wavelength of $\lambda=900 \mathrm{~nm}$. The length of the array in the $z$ direction is $L=7.6 \mathrm{~cm}$. The auxiliary waveguide where light is injected is marked with a white arrow. (e)-(h) Similar to (d), except with increasing the refractive index of the indicated sublattice of the array.

indices of at least $n=2.1$ in $2 \mathrm{D}$ [4, or $n=1.9$ in $3 \mathrm{D}$ 43. In particular, this means that higher order topological BICs could potentially be used to confine light in many important low-index photonic platforms, such as those based on two-photon lithography in photoresist [62, 63] and colloids [64, which are typically composed of materials with refractive indices $n \sim 1.5$. We expect that zero-dimensional bound states in the continuum of the kind described here will lead to an expanded range of devices in which cavity and defect modes, for enhancing light-matter coupling, can be found.

\section{METHODS}

We fabricated our waveguide arrays with a Yb-doped fiber laser (Menlo BlueCut) system emitting circularly polarized sub-picosecond (260 fs) pulse trains at $1030 \mathrm{~nm}$ with a repetition rate of $500 \mathrm{kHz}$. The light was focused inside a borosilicate glass (Corning Eagle XG) sample using an aspheric lens. The borosilicate glass sample was mounted on high-precision $x-y-z$ translation stages (Aerotech). Each individual waveguide was written by translating the glass sample once through the focus of the laser at a speed of $10 \mathrm{~mm} / \mathrm{s}$. This process results in waveguides which only support the fundamental mode, which has an elliptical profile. However, by then orienting the lattice to be 'diagonal' with respect to the surface of the glass slide (i.e. the surface of the glass slide lies along the [1 1 1] direction of the lattice), the coupling constants between neighboring waveguides in both the $\left[\begin{array}{ll}1 & 0\end{array}\right]$ and $\left[\begin{array}{ll}0 & 1\end{array}\right]$ directions are the same, up to fabrication imperfections. By measuring two-waveguide couplers, we determined that our waveguides can be modeled in our full-wave numerical simulations using a Gaussian profile,

$$
\Delta n(\mathbf{r})=\Delta n_{0} e^{-r^{2} / \sigma_{r}^{2}}
$$

with $\Delta n_{0}=2.9 \cdot 10^{-3}$ and $\sigma_{r}=4 \mu \mathrm{m}$. For a separation of $13 \mu \mathrm{m}$ at $\lambda=850 \mathrm{~nm}$, this yields a tight-binding coupling coefficient of $t=1.62 \mathrm{~cm}^{-1}$. For the waveguides used in breaking the chiral symmetry of the lattice as highlighted in Fig. 4, the writing speed was incrementally decreased to be $[8,6,4,2] \mathrm{mm} / \mathrm{s}$ in Fig. $4 \mathrm{~h}-\mathrm{h}$, respectively. These reduced writing speeds can be modeled numerically as shifts of the refractive index of the waveguides of $\Delta n_{0}=$ $[3.1,3.3,3.5,3.7] \cdot 10^{-3}$.

The waveguide arrays were measured using a commercial supercontinuum source (NKT SuperK COMPACT) with a filter to select the desired wavelength (SuperK SELECT). The beam was focused into the sample 
using an aspheric lens with an NA of 0.15 (ThorLabs C280TMD-B) and imaged onto the camera with an achromatic doublet (ThorLabs AC064-015-B-ML). The images were taken using a CMOS camera (ThorLabs DCC1545M).

\section{ACKNOWLEDGMENTS}

This work was supported by the US Office of Naval Research (ONR) Multidisciplinary University Research Initiative (MURI) grant N00014-20-1-2325 on Robust Photonic Materials with High-Order Topological Protection, the ONR Young Investigator Award under grant number N00014-18-1-2595 as well as the Packard Foundation under fellowship number 2017-66821. W.A.B. acknowledges the support of the Eberly Postdoctoral Fellowship at the Pennsylvania State University. M.J. acknowledges the support of the Verne M. Willaman Distinguished Graduate Fellowship at the Pennsylvania State University.

\section{SUPPLEMENTAL INFORMATION FOR: OBSERVATION OF A HIGHER-ORDER TOPOLOGICAL BOUND STATE IN THE CONTINUUM}

\section{HIGHER ORDER BOUND STATE IN THE CONTINUUM WITHOUT SEPARABILITY}

One known mechanism for creating bound states in the continuum (BICs) is through separability 34, in which the Hamiltonian of the system can be divided into two (or more) parts,

$$
\hat{H}(\mathbf{r})=\hat{H}_{x}(x)+\hat{H}_{y}(y)
$$

that only depend on a single spatial coordinate. Then, by finding a localized bound state of each individual portion, for example, $H_{x}(x) \psi_{n}(x)=E_{n}^{(x)} \psi_{n}(x)$ and $H_{y} \phi_{m}(y)=E_{m}^{(y)} \phi_{m}(y)$, the combined state, $\psi_{n} \phi_{m}$, is a bound state of $\hat{H}(\mathbf{r})$ with energy $E_{n}^{(x)}+E_{m}^{(y)}$, which may reside within the continuum $\hat{H}(\mathbf{r})$. Furthermore, one may suspect that this is the origin of the BIC we report in the main text, as the tight-binding model corresponding to our waveguide arrays is separable.

(a)

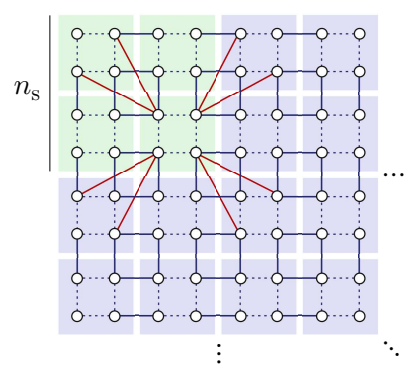

(b)

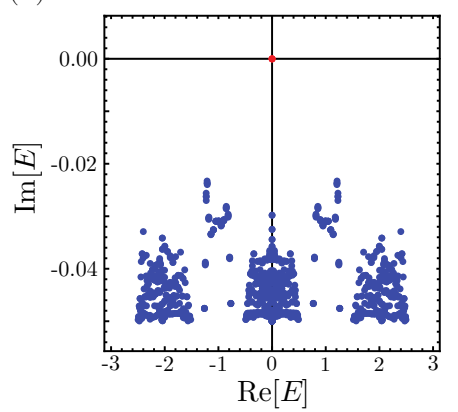

(c) $(\mathrm{d})$

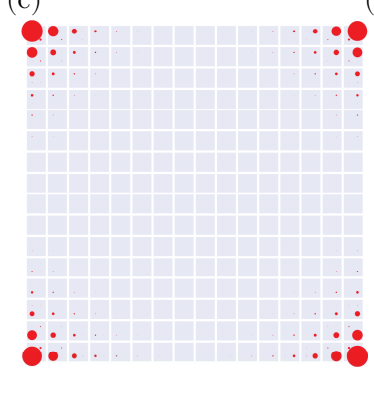

(d)

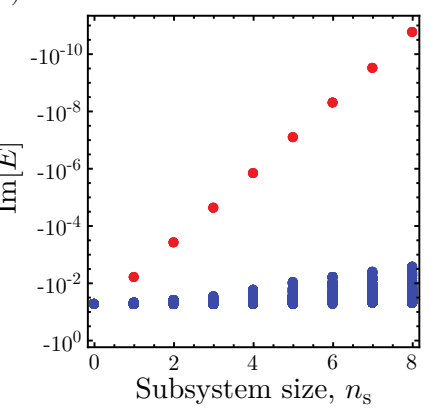

FIG. S1. (a) Schematic depicting the tight-binding model used as an example of a non-separable higher-order topological BIC. Dashed lines indicate couplings of $t_{\text {intra }}=0.25$, solid black lines indicate couplings of $t_{\text {inter }}=1$, and solid red lines indicate chiral-preserving non-separable couplings, $t=0.025$. Only a single set of chiral-preserving non-separable couplings are shown for simplicity. Unit cells are indicated by shaded regions behind sets of nodes. Green unit cells are part of the 'subsystem,' while blue unit cells are part of the 'environment,' and have an added on-site loss of $\gamma=0.05$. Only a single corner of the lattice is shown, but all four corners are part of the subsystem for these simulations, and do not have any on-site loss. (b) Eigenvalues for this lattice shown in the complex plane for $n_{\mathrm{s}}=3$, i.e. the total number of unit cells in each corner which constitute the 'subsystem' is $3 \times 3$. The lattice has $n_{1}=16$ unit cells along its sides, for a total lattice size of $16 \times 16$. (c) The probability densities of the four eigenstates corresponding to the BICs from (b). (d) Plot of the decay rate of the eigenvalues of the lattice as $n_{\mathrm{s}}$ is increased.

However, this is not the origin of the protection of the BICs that we observe in our waveguide arrays [41]. To demonstrate this explicitly, in Fig. S1 we show numerical tight-binding calculations of a higher-order topological insulator that obeys both of the necessary protecting symmetries of the BIC, $C_{4 v}$ and chiral symmetry, but breaks separability. This lattice is schematically shown in Fig. S1a. We then divide this finite lattice into two regions, the 'subsystem,' a region of $n_{\mathrm{s}}$ unit cells next to each of the four corners of the lattice, and the 'environment,' which are 

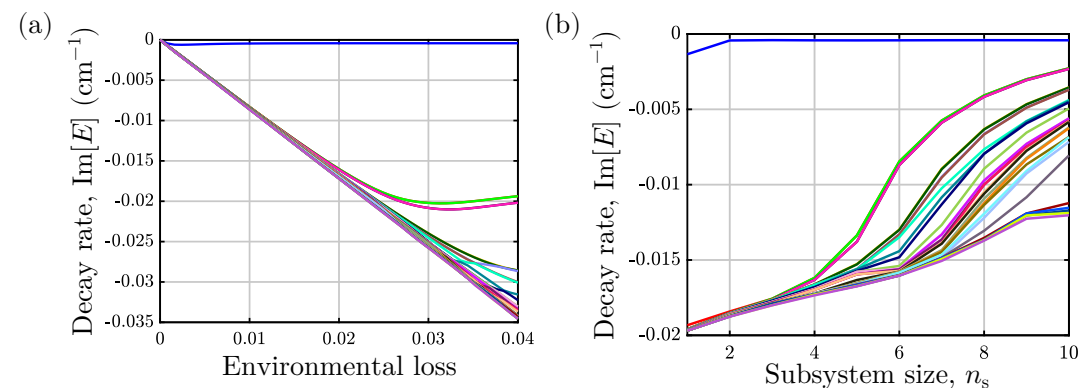

FIG. S2. (a) Plot of the decay rates of the 30 least lossy eigenvalues of the lattice as a function of the added loss, $\gamma$, to the waveguides in the environment. The plateau seen in the decay rate of the corner-localized mode corresponds to its true radiative rate in an infinite lattice. Here, $n_{1}=32$ (i.e. the total lattice size is $32 \times 32$ ), and $n_{\mathrm{s}}=4$. (b) Plot of the decay rates of the 30 least lossy eigenvalues of the full lattice as a function of the edge length of the device region, $n_{\mathrm{s}}$ for $\gamma=0.02$ and $n_{1}=32$. In both (a) and (b), the decay rate of the corner-localized mode is reaching a nearly constant value at approximately $\operatorname{Im}[E]=-4 \cdot 10^{-4} \mathrm{~cm}^{-1}$.

the remainder of the unit cells in the lattice. A small, but non-zero, amount of loss is then added to the environment to simulate radiation loss, $0<\gamma \ll 1$. As can be seen in Fig. S1b, this lattice still possesses four essentially real eigenvalues, whose corresponding eigenstates are exponentially localized to the corners of the lattice, see Fig. S1;. Finally, by changing the size of the device region, we can confirm that the energies of these corner-localized states exponentially converge to be real, confirming that these are BICs, not resonances, as shown in Fig. S1 d.

Thus, even though the waveguide array that we study in the main text is separable, this separability is not what protects the BICs that we observe in our array. Even in the absence of separability, our arrays would still exhibit BICs, so long as the two necessary protecting symmetries, $C_{4 v}$ and chiral, were preserved.

\section{EFFECT OF NEXT-NEAREST-NEIGHBOR COUPLINGS IN THE WAVEGUIDE ARRAY}

As discussed in the main text, in our waveguide arrays it is impossible to completely remove next-nearest-neighbor couplings between waveguides as the coupling strength is an exponentially decaying function of the spatial separation between the waveguides, $t(\lambda)=e^{-\alpha(\lambda) l}$, in which $\alpha(\lambda)$ is a wavelength-dependent constant. In particular, this means that the couplings between diagonally adjacent waveguides in our arrays will be non-vanishing, and break chiral symmetry, placing a practical limit on the decay length of the corner modes. However, in practice, the coupling coefficients corresponding to this process are small relative to the dominant energy scale in the array, i.e. the larger of $t_{\text {intra }}$ or $t_{\text {inter }}$ depending on whether the lattice is in the topological phase. For example, for the topological lattice shown in Fig. $2 \mathrm{~d}$,f of the main text, $t_{\text {diag }} / t_{\text {inter }} \sim 0.08$, where $t_{\text {diag }}$ is the next-neighbor coupling strength between waveguides across the diagonal in the unit cell.

Moreover, we can calculate the effect of this coupling constant on the now-finite decay length of the corner-localized mode using a tight-binding model with a small amount of non-Hermitian loss added to an 'environment' region. Mathematically, this corresponds to

$$
\hat{H}_{\text {tot }}=\hat{H}-\sum_{j \in \text { env. wgs. }} i \gamma|j\rangle\langle j|,
$$

in which $\hat{H}$ is given by Eq. 1 of the main text, and in which the sum runs over those waveguides in the environment. As can be seen in Fig. [S2 a, the decay length of the corner-localized mode quickly saturates as a function of the added loss to the environment, and this plateau corresponds to the radiative loss of the corner-localized resonance in an infinite lattice without added loss by the Limiting Absorption Principle 65 68. Moreover, we can confirm this decay length of the corner-localized mode by fixing the added environmental loss and varying the size of the device region, shown in Fig. $\mathrm{S} 2 \mathrm{~b}$. In both cases, the decay length converges to approximately $\operatorname{Im}[E]=-4 \cdot 10^{-4} \mathrm{~cm}^{-1}$ As stated in the Main Text, for the topological lattice shown in Fig. 2d,f, the decay length is $L_{\text {decay }} \sim 25 \mathrm{~m}$, which is significantly longer than the waveguide arrays in our experiment $(L=7.6 \mathrm{~cm})$. As such, for the purposes of our experiment, our waveguide array is effectively chiral symmetric.

[1] Klitzing, K. v., Dorda, G. \& Pepper, M. New Method for High-Accuracy Determination of the Fine-Structure Con- 
Lett. 45, 494-497 (1980). URL https://link.aps .org/ doi/10.1103/PhysRevLett.45.494

[2] Halperin, B. Quantized Hall Conductance, CurrentCarrying Edge States, and the Existence of Extended States in a Two-Dimensional Disordered Potential. Phys. Rev. B 25, 2185-2190 (1982).

[3] Thouless, D. J., Kohmoto, M., Nightingale, M. P. \& den Nijs, M. Quantized Hall Conductance in a TwoDimensional Periodic Potential. Phys. Rev. Lett. 49, 405-408 (1982). URL https://link.aps.org/doi/10. 1103/PhysRevLett.49.405.

[4] Büttiker, M. Absence of backscattering in the quantum Hall effect in multiprobe conductors. Phys. Rev. $B$ 38, 9375-9389 (1988). URL https://link.aps.org/ doi/10.1103/PhysRevB.38.9375

[5] Haldane, F. D. M. Model for a Quantum Hall Effect without Landau Levels: Condensed-Matter Realization of the "Parity Anomaly". Phys. Rev. Lett. 61, 20152018 (1988). URL http://link.aps.org/doi/10.1103/ PhysRevLett.61.2015

[6] Haldane, F. D. M. \& Raghu, S. Possible Realization of Directional Optical Waveguides in Photonic Crystals with Broken Time-Reversal Symmetry. Phys. Rev. Lett. 100, 013904 (2008). URL https://link.aps.org/doi/ 10.1103/PhysRevLett.100.013904

[7] Wang, Z., Chong, Y., Joannopoulos, J. D. \& Soljačić, M. Observation of unidirectional backscattering-immune topological electromagnetic states. Nature 461, 772775 (2009). URL https://www.nature.com/nature/ journal/v461/n7265/full/nature08293.html.

[8] Umucalılar, R. O. \& Carusotto, I. Artificial gauge field for photons in coupled cavity arrays. Phys. Rev. A 84, 043804 (2011). URL https://link.aps.org/doi/10. 1103/PhysRevA.84.043804

[9] Hafezi, M., Demler, E. A., Lukin, M. D. \& Taylor, J. M. Robust optical delay lines with topological protection. Nat. Phys. 7, 907-912 (2011).

[10] Fang, K., Yu, Z. \& Fan, S. Realizing effective magnetic field for photons by controlling the phase of dynamic modulation. Nat. Photon. 6, 782-787 (2012).

[11] Kitagawa, T. et al. Observation of topologically protected bound states in photonic quantum walks. Nat. Commun. 3, 1872 (2012). URL https://www.nature. com/articles/ncomms1872.

[12] Rechtsman, M. C. et al. Photonic Floquet topological insulators. Nature 496, 196-200 (2013). URL http://www.nature.com/nature/journal/v496/ n7444/abs/nature12066.html.

[13] Khanikaev, A. B. et al. Photonic topological insulators. Nat. Mater. 12, 233-239 (2013). URL https://www.nature.com/nmat/journal/v12/n3/full/ nmat3520.html.

[14] Hafezi, M., Mittal, S., Fan, J., Migdall, A. \& Taylor, J. M. Imaging topological edge states in silicon photonics. Nat. Photon. 7, 1001-1005 (2013). URL http://www . nature.com/doifinder/10.1038/nphoton.2013.274

[15] Su, W. P., Schrieffer, J. R. \& Heeger, A. J. Solitons in Polyacetylene. Phys. Rev. Lett. 42, 1698-1701 (1979).

[16] King-Smith, R. \& Vanderbilt, D. Theory of polarization of crystalline solids. Physical Review B 47, 1651 (1993).

[17] Zak, J. Berrys phase for energy bands in solids. Physical review letters 62, 2747 (1989).

[18] Benalcazar, W. A., Teo, J. C. Y. \& Hughes, T. L. Classification of two-dimensional topological crystalline su- perconductors and Majorana bound states at disclinations. Phys. Rev. B 89, 224503 (2014). URL https: //link.aps.org/doi/10.1103/PhysRevB.89.224503.

[19] Benalcazar, W. A., Bernevig, B. A. \& Hughes, T. L. Quantized electric multipole insulators. Science 357, 61-66 (2017). URL http://science.sciencemag.org/ content/357/6346/61

[20] Benalcazar, W. A., Bernevig, B. A. \& Hughes, T. L. Electric multipole moments, topological multipole moment pumping, and chiral hinge states in crystalline insulators. Phys. Rev. B 96, 245115 (2017). URL https: //link.aps.org/doi/10.1103/PhysRevB.96.245115.

[21] Song, Z., Fang, Z. \& Fang, C. $(d-2)$-dimensional Edge States of Rotation Symmetry Protected Topological States. Phys. Rev. Lett. 119, 246402 (2017). URL https://link.aps.org/doi/10.1103/ PhysRevLett.119.246402.

[22] Langbehn, J., Peng, Y., Trifunovic, L., von Oppen, F. \& Brouwer, P. W. Reflection-Symmetric Second-Order Topological Insulators and Superconductors. Phys. Rev. Lett. 119, 246401 (2017). URL https://link.aps. org/doi/10.1103/PhysRevLett.119.246401| Publisher: American Physical Society.

[23] Schindler, F. et al. Higher-order topological insulators. Science Advances 4, eaat0346 (2018). URL https://advances.sciencemag.org/content/4/ 6/eaat0346 Publisher: American Association for the Advancement of Science Section: Research Article.

[24] Zilberberg, O. et al. Photonic topological boundary pumping as a probe of $4 \mathrm{~d}$ quantum hall physics. Nature 553, 59-62 (2018).

[25] Wieder, B. J. \& Bernevig, B. A. The axion insulator as a pump of fragile topology. arXiv:1810.02373 (2018).

[26] van Miert, G. \& Ortix, C. Higher-order topological insulators protected by inversion and rotoinversion symmetries. Phys. Rev. B 98, 081110 (2018). URL https: //link.aps.org/doi/10.1103/PhysRevB.98.081110.

[27] Ezawa, M. Minimal models for wannier-type higher-order topological insulators and phosphorene. Phys. Rev. B 98, 045125 (2018). URL https://link.aps.org/doi/ 10.1103/PhysRevB.98.045125

[28] Benalcazar, W. A., Li, T. \& Hughes, T. L. Quantization of fractional corner charge in $C_{n}$-symmetric higherorder topological crystalline insulators. Phys. Rev. B 99, 245151 (2019). URL https://link.aps.org/doi/ 10.1103/PhysRevB.99.245151

[29] Lee, E., Kim, R., Ahn, J. \& Yang, B.-J. Higherorder band topology and corner charges in monolayer graphdiyne. arXiv preprint arXiv:1904.11452 (2019).

[30] Sheng, X.-L. et al. Two-dimensional second-order topological insulator in graphdiyne. arXiv preprint arXiv:1904.09985 (2019).

[31] Schindler, F. et al. Fractional corner charges in spinorbit coupled crystals. arXiv preprint arXiv:190\%.10607 (2019).

[32] Petrides, I. \& Zilberberg, O. Higher-order topological insulators, topological pumps and the quantum Hall effect in high dimensions. Phys. Rev. Research 2, 022049 (2020). URL https://link.aps.org/doi/10. 1103/PhysRevResearch.2.022049 Publisher: American Physical Society.

[33] von Neumann, J. \& Wigner, E. Über merkwürdige diskrete eigenwerte. Phys. Z. 30, 465 (1929).

[34] Hsu, C. W., Zhen, B., Stone, A. D., Joannopoulos, J. D. 
\& Soljačić, M. Bound states in the continuum. Nat. Rev. Mater. 1, 16048 (2016). URL http://www.nature.com/ articles/natrevmats201648.

[35] Friedrich, H. \& Wintgen, D. Interfering resonances and bound states in the continuum. Phys. Rev. A 32, 3231-3242 (1985). URL https://link.aps.org/doi/ 10.1103/PhysRevA.32.3231

[36] Plotnik, Y. et al. Experimental Observation of Optical Bound States in the Continuum. Phys. Rev. Lett. 107, 183901 (2011). URL https://link.aps.org/doi/ 10.1103/PhysRevLett.107.183901

[37] Weimann, S. et al. Compact Surface Fano States Embedded in the Continuum of Waveguide Arrays. Phys. Rev. Lett. 111, 240403 (2013). URL https://link.aps. org/doi/10.1103/PhysRevLett.111.240403.

[38] Hsu, C. W. et al. Observation of trapped light within the radiation continuum. Nature 499, 188-191 (2013). URL http://www.nature.com/doifinder/10. 1038/nature12289

[39] Zhou, H. et al. Perfect single-sided radiation and absorption without mirrors. Optica 3, 1079-1086 (2016). URL https://www . osapublishing.org/optica/ abstract.cfm?uri=optica-3-10-1079.

[40] Cerjan, A., Hsu, C. W. \& Rechtsman, M. C. Bound States in the Continuum through Environmental Design. Phys. Rev. Lett. 123, 023902 (2019). URL https:// link.aps.org/doi/10.1103/PhysRevLett.123.023902

[41] Benalcazar, W. A. \& Cerjan, A. Bound states in the continuum of higher-order topological insulators. Phys. Rev. B 101, 161116 (2020). URL https://link.aps. org/doi/10.1103/PhysRevB.101.161116

[42] Joannopoulos, J. D., Johnson, S. G., Winn, J. N. \& Meade, R. D. Photonic Crystals: Molding the Flow of Light (Second Edition) (Princeton University Press, 2011).

[43] Men, H., Lee, K. Y. K., Freund, R. M., Peraire, J. \& Johnson, S. G. Robust topology optimization of three-dimensional photonic-crystal bandgap structures. Opt. Express 22, 22632 (2014). URL https://www.osapublishing.org/oe/abstract. cfm?uri=oe-22-19-22632.

[44] Cerjan, A. \& Fan, S. Complete photonic band gaps in supercell photonic crystals. Phys. Rev. A 96, 051802 (2017). URL https://link.aps.org/doi/10. 1103/PhysRevA.96.051802

[45] Peterson, C. W., Benalcazar, W. A., Hughes, T. L. \& Bahl, G. A quantized microwave quadrupole insulator with topologically protected corner states. Nature $\mathbf{5 5 5}$, 346 EP - (2018). URL http://dx.doi.org/10.1038/ nature25777.

[46] Noh, J. et al. Topological protection of photonic mid-gap defect modes. Nature Photonics (2018). URL https: //doi.org/10.1038/s41566-018-0179-3

[47] Mittal, S. et al. Photonic quadrupole topological phases. Nature Photonics 13, 692-696 (2019). URL https:// www.nature.com/articles/s41566-019-0452-0 Number: 10 Publisher: Nature Publishing Group.

[48] Li, M. et al. Higher-order topological states in photonic kagome crystals with long-range interactions. $\mathrm{Na}$ ture Photonics 14, 89-94 (2020). URL https://www. nature.com/articles/s41566-019-0561-9. Number: 2 Publisher: Nature Publishing Group.

[49] Serra-Garcia, M. et al. Observation of a phononic quadrupole topological insulator. Nature 555, 342-345
(2018)

[50] Ni, X., Weiner, M., Alù, A. \& Khanikaev, A. B. Observation of higher-order topological acoustic states protected by generalized chiral symmetry. Nature Materials 18, 113 (2019). URL https://www.nature.com/articles/ s41563-018-0252-9

[51] Xue, H., Yang, Y., Gao, F., Chong, Y. \& Zhang, B. Acoustic higher-order topological insulator on a kagome lattice. Nature Materials 18, 108-112 (2019). URL https://www.nature.com/articles/ s41563-018-0251-x Number: 2 Publisher: Nature Publishing Group.

[52] Xue, H. et al. Realization of an Acoustic ThirdOrder Topological Insulator. Phys. Rev. Lett. 122, 244301 (2019). URL https://link.aps.org/doi/10. 1103/PhysRevLett.122.244301. Publisher: American Physical Society.

[53] Ni, X., Li, M., Weiner, M., Alù, A. \& Khanikaev, A. B. Demonstration of a quantized acoustic octupole topological insulator. arXiv:1911.06469 [condmat, physics:physics] (2019). URL http://arxiv.org/ abs/1911.06469. ArXiv: 1911.06469.

[54] Xue, H. et al. Observation of an acoustic octupole topological insulator. Nature Communications 11, 2442 (2020). URL https://www.nature.com/articles/ s41467-020-16350-1. Number: 1 Publisher: Nature Publishing Group.

[55] Imhof, S. et al. Topolectrical-circuit realization of topological corner modes. Nature Physics 14, 925929 (2018). URL https://www.nature.com/articles/ s41567-018-0246-1

[56] Bao, J. et al. Topoelectrical circuit octupole insulator with topologically protected corner states. Phys. Rev. $B$ 100, 201406 (2019). URL https://link.aps.org/ doi/10.1103/PhysRevB.100.201406. Publisher: American Physical Society.

[57] Kempkes, S. N. et al. Robust zero-energy modes in an electronic higher-order topological insulator. $\mathrm{Na}$ ture Materials 18, 1292-1297 (2019). URL https:// www. nature.com/articles/s41563-019-0483-4 Number: 12 Publisher: Nature Publishing Group.

[58] Davis, K. M., Miura, K., Sugimoto, N. \& Hirao, K. Writing waveguides in glass with a femtosecond laser. Opt. Lett. 21, 1729-1731 (1996). URL https://www.osapublishing.org/ol/abstract. cfm?uri=ol-21-21-1729. Publisher: Optical Society of America.

[59] Szameit, A. \& Nolte, S. Discrete optics in femtosecondlaser-written photonic structures. J. Phys. B: At. Mol. Opt. Phys. 43, 163001 (2010). URL http://stacks .iop. org $/ 0953-4075 / 43 / i=16 / a=163001$

[60] Liu, F. \& Wakabayashi, K. Novel Topological Phase with a Zero Berry Curvature. Phys. Rev. Lett. 118, 076803 (2017). URL http://link.aps.org/doi/10. 1103/PhysRevLett.118.076803

[61] Chen, Z.-G., Xu, C., Al Jahdali, R., Mei, J. \& Wu, Y. Corner states in a second-order acoustic topological insulator as bound states in the continuum. Phys. Rev. B 100, 075120 (2019). URL https://link.aps.org/doi/ 10.1103/PhysRevB.100.075120

[62] Freymann, G. v. et al. Three-Dimensional Nanostructures for Photonics. Advanced Functional Materials 20, 1038-1052 (2010). URL https://onlinelibrary. wiley.com/doi/abs/10.1002/adfm.200901838 
63] Bückmann, T. et al. Tailored 3D Mechanical Metamaterials Made by Dip-in Direct-Laser-Writing Optical Lithography. Advanced Materials 24, 2710-2714 (2012). URL https://onlinelibrary.wiley.com/doi/abs/10. 1002/adma. 201200584

[64] Norris, D. J., Arlinghaus, E. G., Meng, L., Heiny, R. \& Scriven, L. E. Opaline Photonic Crystals: How Does Self-Assembly Work? Advanced Materials 16, 1393-1399 (2004). URL https://onlinelibrary.wiley.com/doi/ abs/10.1002/adma.200400455.

[65] v. Ignatowsky, W. Reflexion elektromagnetisches Wellen an einem Draht. Ann. Phys. 323, 495-522 (1905).
URL http://onlinelibrary.wiley.com/doi/10.1002/ andp.19053231305/abstract

[66] Eidus, D. M. On the principle of limiting absorption. Mat. Sb. (N.S.) 57(99), 13-44 (1962).

[67] Schulenberger, J. R. \& Wilcox, C. H. The limiting absorption principle and spectral theory for steady-state wave propagation in inhomogeneous anisotropic media. Arch. Rational Mech. Anal. 41, 46-65 (1971). URL http: //link.springer.com/article/10.1007/BF00250177.

[68] Cerjan, A. \& Stone, A. D. Why the laser linewidth is so narrow: a modern perspective. Phys. Scr. 91, 013003 (2016). URL http://stacks .iop.org/1402-4896/91/i= $1 / \mathrm{a}=013003$. 